\title{
Demonstrations of a Pair of Theorems in Geometry.
}

\author{
By H. F. Buichfeldt.
}

I.

If , in any triangle $A B C$, the angle $A>$ the angle $B$, and lines $A D$ and $B E$ be drawn so that the angles $B A D$ and $D A C$ are, respectively, greater than the angles $A B E$ and $E B C$, then is $B E>A D$. (Fig. 6.)

Proof: Draw $\mathrm{AD}^{\prime}$ and $\mathrm{AD}^{\prime \prime}$ making angles $\mathrm{BAD}^{\prime}$ and $\mathrm{D}^{\prime \prime} \mathrm{AC}$, respectively, equal to the angles $\mathrm{ABE}$ and $\mathrm{EBC}$.

Then we can show that $\mathrm{AD}^{\prime}$ and $\mathrm{AD}^{\prime \prime}$ are each less than $\mathrm{BE}$.

First, by placing the triangle $\mathrm{ABD}^{\prime}$ on the triangle $\mathrm{BAE}$ so that $A B$ and the angle $B A D^{\prime}$ coincide respectively with $B A$ and the angle $\mathrm{ABE}$, we find, since angle $\mathrm{A}>$ angle $\mathrm{B}, \mathrm{AD}^{\prime}<\mathrm{BE}$.

Second, by placing the triangle $\mathrm{ACD}^{\prime \prime}$ on the triangle $\mathrm{BCE}$ so that the angles $\mathrm{C}$ in both triangles coincide, and the side $\mathrm{CA}$ falls along $\mathrm{CB}(\mathrm{AC}<\mathrm{BC})$, the lines $\mathrm{AD}^{\prime \prime}$ and $\mathrm{BE}$ will be parallel. Draw $A F$ parallel to $\mathrm{CE}$, and we find $A D^{\prime \prime}=\mathrm{FE}<\mathrm{BE}$.

Now, of the three lines $A D^{\prime}, A D$, and $A D^{\prime \prime}$, the one which is the most remote from the perpendicular, drawn from $A$ upon $B C$, and consequently the greatest, must be one of the lines $\mathrm{AD}^{\prime}$ and $\mathrm{AD}^{\prime \prime}$, which lie on opposite sides of AD.

Hence, $A D$, being less than either $A D^{\prime}$ or $A D^{\prime \prime}$, which are each less than $\mathrm{BE}$, must itself be less than $\mathrm{BE}$.

Cor. If the angles $\mathrm{ABE}$ and $\mathrm{EBC}$ are, respectively, not greater than the angles $\mathrm{BAD}$ and $\mathrm{DAC}$, and if $\mathrm{AD}=\mathrm{BE}$, it follows that the angle $A=$ the angle $B$, and that these angles are divided in the same ratio by $\mathrm{AD}$ and $\mathrm{BE}$.

Hence, if the angles $A$ and $B$ are bisected by $A D$ and $B E$, and these lines are equal, the triangle $\mathrm{ABC}$ is isoceles. 
II.

If, in any triangle $A B C, B C>A C$, and lines $A D$ and $B E$ be drawn so that $C D$ and $D B$ are, respectively, greater than $C E$ and $E A$, then is $B E>A D$. (Fig. 7.)

Proof: Draw $\mathrm{AD}^{\prime \prime}$ and $\mathrm{AD}^{\prime}$ making $\mathrm{BD}^{\prime}$ and $\mathrm{D}^{\prime \prime} \mathrm{C}$, respectively equal to $\mathrm{AE}$ and $\mathrm{EC}$.

Then we can show that $\mathrm{AD}^{\prime \prime}$ and $\mathrm{AD}^{\prime}$ are each less than $\mathrm{BE}$.

First, the triangles $A D^{\prime} B$ and $B E A$ have two sides of the one respectively equal to two sides of the other, but the included angles $A$ and $B$ unequal (since $B C>A C$ ). As the angle $A>$ the angle $B$, we find $B E>A D^{\prime}$.

Second, the two triangles $\mathrm{ACD}^{\prime \prime}$ and $\mathrm{BCE}$ have the angle $\mathrm{C}$ common, $\mathrm{CD}^{\prime \prime}=\mathrm{CE}$, and $\mathrm{BC}>\mathrm{AC}$. Therefore the angle $\mathrm{CEB}>$ the angle $\mathrm{CD}^{\prime \prime} \mathrm{A}$. Hence, the angle $\mathrm{CAD}^{\prime \prime}>$ the angle $\mathrm{CBE}$. Also, the supplement of the angle $\mathrm{CAD}^{\prime \prime}>$ the angle $\mathrm{CBE}$, since the supplement of the angle $A>$ the angle $B$.

Now, place the triangle $\mathrm{CAD}^{\prime \prime}$ on the triangle $\mathrm{CBE}$ so that the angles $\mathrm{C}$ in both triangles coincide, and $\mathrm{CE}$ coincides with $\mathrm{CD}^{\prime \prime}$. Then, as each angle at $A$ bas been shown to be greater than the angle CBE, the oblique EB must be greater than EA.

Thus, $\mathrm{EB}$ is greater than both $\mathrm{AD}^{\prime}$ and $\mathrm{AD}^{\prime \prime}$, one of which is, as shown in (I.), greater than $A D$. Therefore, $E B>A D$.

Cor. If $\mathrm{CD}$ and $\mathrm{DB}$ are, respectively, not less than $\mathrm{CE}$ and $\mathrm{EA}$, and $A D=B E$, it follows that $A C=B C$, and these sides are divided in the same ratio by $A D$ and $B E$.

Hence, if two medians of a triangle are equal, the triangle is isosceles. 